\title{
741.
}

\section{ON A THEOREM OF ABEL'S RELATING TO A QUINTIC EQUATION.}

[From the Proceedings of the Cambridge Philosophical Society, vol. III. (1880), pp. 155-159.]

The theorem in question is given, Euvres Complètes, [Christiania, 1881], t. II., p. 266, as an extract from a letter to Crelle dated 14th March, 1826, as follows:

"Si une équation du cinquième degré dont les coefficients sont des nombres rationnels est résoluble algébriquement, on peut donner aux racines la forme suivante:

$$
x=c+A a^{\frac{1}{5}} a_{1}^{\frac{2}{5}} a_{2}^{\frac{4}{5}} a_{3}^{\frac{3}{5}}+A_{1} a_{1}^{\frac{1}{5}} a_{2}^{\frac{2}{5}} a_{3}^{\frac{4}{5}} a^{\frac{5}{5}}+A_{2} a_{2}^{\frac{1}{5}} a_{3}^{\frac{2}{5}} a^{\frac{4}{5}} a_{1}^{\frac{3}{5}}+A_{3} a_{3}^{\frac{1}{5}} a^{\frac{2}{5}} a_{1}^{\frac{4}{5}} a_{2}^{\frac{3}{5}},
$$

où

$$
\begin{aligned}
& a=m+n \sqrt{ }\left(1+e^{2}\right)+\sqrt{ }\left[h\left(1+e^{2}+\sqrt{ }\left(1+e^{2}\right)\right)\right], \\
& a_{1}=m-n \sqrt{ }\left(1+e^{2}\right)+\sqrt{ }\left[h\left(1+e^{2}-\sqrt{ }\left(1+e^{2}\right)\right)\right] \text {, } \\
& a_{2}=m+n \sqrt{ }\left(1+e^{2}\right)-\sqrt{ }\left[h\left(1+e^{2}+\sqrt{ }\left(1+e^{2}\right)\right)\right] \text {, } \\
& a_{3}=m-n \sqrt{ }\left(1+e^{2}\right)-\sqrt{ }\left[h\left(1+e^{2}-\sqrt{ }\left(1+e^{2}\right)\right)\right], \\
& A=K+K^{\prime} a+K^{\prime \prime} a_{2}+K^{\prime \prime \prime} a a_{2}, \quad A_{1}=K+K^{\prime} a_{1}+K^{\prime \prime} a_{3}+K^{\prime \prime \prime} a_{1} a_{3} \text {, } \\
& A_{2}=K+K^{\prime} a_{2}+K^{\prime \prime} a+K^{\prime \prime \prime} a a_{2}, \quad A_{3}=K+K^{\prime} a_{3}+K^{\prime \prime} a_{1}+K^{\prime \prime \prime} a_{1} a_{3} .
\end{aligned}
$$

Les quantités $c, h, e, m, n, K, K^{\prime}, K^{\prime \prime}, K^{\prime \prime \prime}$ sont des nombres rationnels. Mais de cette manière l'équation $x^{5}+a x+b=0$ n'est pas résoluble tant que $a$ et $b$ sont des quantités quelconques. J'ai trouvé de pareils théorèmes pour les équations du $7^{\text {ème, }}$, $11^{\text {ème }}, 13^{\text {ème }}$, etc. degré."

It is easy to see that $x$ is the root of a quintic equation, the coefficients of which are rational and integral functions of $a, a_{1}, a_{2}, a_{3}$ : these coefficients are not symmetrical functions of $a, a_{1}, a_{2}, a_{3}$, but they are functions which remain unaltered 
by the cyclical change $a$ into $a_{1}, a_{1}$ into $a_{2}, a_{2}$ into $a_{3}, a_{3}$ into $a$. But the coefficients of the quintic equation must be rational functions of $c, h, e, m, n, K, K^{\prime}, K^{\prime \prime}, K^{\prime \prime \prime}$ : hence regarding $a, a_{1}, a_{2}, a_{3}$, as the roots of a quartic equation, the coefficients of this equation being rational functions of $m, n, e, h$, this equation must be such that every rational function of the roots, unchanged by the aforesaid cyclical change of the roots, shall be rationally expressible in terms of these quantities $m, n, e, h$ : or, what is the same thing, the group of the quartic equation, using the term "group of the equation" in the sense assigned to it by Galois, must be $a a_{1} a_{2} a_{3}, a_{1} a_{2} a_{3} a$, $a_{2} a_{3} a a_{1}, a_{3} a a_{1} a_{2}$. And conversely, the quartic equation being of this form, $x$ will be the root of a quintic equation, the coefficients whereof are rational and integral functions of $c, h, e, m, n, K, K^{\prime}, K^{\prime \prime}, K^{\prime \prime \prime}$.

To investigate the form of a quartic equation having the property just referred to, let it be proposed to find $\gamma, \gamma^{\prime}$ functions of $e, h$, such that $\gamma^{2}+\gamma^{\prime 2}$ is a rational function of $e, h$, but that $\gamma^{2}-\gamma^{\prime 2}, \gamma \gamma^{\prime}$ are rational multiples of the same quadric radical $\sqrt{\theta}$. Assume that we have

then

$$
\gamma^{2}-\gamma^{\prime 2}=2 p \sqrt{\theta}, \quad \gamma \gamma^{\prime}=q \sqrt{\theta}
$$

$$
\left(\gamma^{2}+\gamma^{\prime 2}\right)^{2}=4\left(p^{2}+q^{2}\right) \theta
$$

that $\gamma^{2}+\gamma^{\prime 2}$ may be rational, we must have $p^{2}+q^{2}=\lambda^{2} \theta$, or say $p^{2}+q^{2}=h^{2} \theta$; hence, $\theta=\frac{p^{2}}{h^{2}}+\frac{q^{2}}{h^{2}}$ must be a sum of two squares, or, assuming one of these equal to unity and the other of them equal to $e^{2}$, say $\theta=1+e^{2}$, we satisfy the required equation by taking $p=h, q=h e$ : viz. we thus have

$$
\gamma^{2}-\gamma^{\prime 2}=2 h \sqrt{1+e^{2}}, \quad \gamma \gamma^{\prime}=h e \sqrt{1+e^{2}}, \quad \gamma^{2}+\gamma^{\prime 2}=2 h\left(1+e^{2}\right) ;
$$

and thence also

$$
\gamma^{2}=h\left(1+e^{2}+\sqrt{1+e^{2}}\right), \quad \gamma^{\prime 2}=h\left(1+e^{2}-\sqrt{1+e^{2}}\right),
$$

the roots of these expressions, or values of $\gamma, \gamma^{\prime}$, being such that

$$
\gamma \gamma^{\prime}=h e \sqrt{1+e^{2}} \text {. }
$$

Taking now $\alpha$ rational, $=m$ suppose, and $\beta$ a rational multiple of

$$
\sqrt{1+e^{2}},=h \sqrt{1+e^{2}},
$$

suppose; it is easy to see that the quartic equation which has for its roots

$$
a, a_{1}, a_{2}, a_{3}=\alpha+\beta+\gamma, \alpha-\beta+\gamma^{\prime}, \alpha+\beta-\gamma, \alpha-\beta-\gamma^{\prime},
$$

has the property in question, viz. that every rational function of the roots unchangeable by the cyclical change $a$ into $a_{1}, a_{1}$ into $a_{2}, a_{2}$ into $a_{3}, a_{3}$ into $a$, is rationally expressible in terms of $e, h, m, n$.

It will be sufficient to give the proof in the case of a rational and integral function; such a function, unchangeable as aforesaid, is of the form

$$
\phi\left(a, a_{1}, a_{2}, a_{3}\right)+\phi\left(a_{1}, a_{2}, a_{3}, a\right)+\phi\left(a_{2}, a_{3}, a, a_{1}\right)+\phi\left(a_{3}, a, a_{1}, a_{2}\right) ;
$$


and if $\phi\left(\alpha, a_{1}, a_{2}, a_{3}\right)$ contains a term $\alpha^{m} \beta^{n} \gamma^{p} \gamma^{\prime q}$, then the other three functions will contain respectively the terms

$$
\alpha^{m}(-\beta)^{n} \gamma^{\prime p}(-\gamma)^{q}, \quad \alpha^{m} \beta^{n}(-\gamma)^{p}\left(-\gamma^{\prime}\right)^{q}, \quad \alpha^{m}(-\beta)^{n}\left(-\gamma^{\prime}\right)^{p}(\gamma)^{q}
$$

viz. the sum of the four terms is

$$
=\alpha^{m} \beta^{n}\left[\left\{1+(-)^{p+q} 1\right\} \gamma^{p} \gamma^{\prime q}+\left\{(-)^{n+p} 1+(-)^{n+q} 1\right\} \gamma^{q} \gamma^{\prime p}\right] .
$$

This obviously vanishes unless $p$ and $q$ are both even, or both odd; and the cases to be considered are $1^{\circ}, n$ even, $p$ and $q$ even; $2^{\circ}, n$ odd, $p$ and $q$ even; $3^{\circ}, n$ even, $p$ and $q$ odd; $4^{\circ}, n$ odd, $p$ and $q$ odd. Writing, for greater distinctness, $2 n$ or $2 n+1$ for $n$, according as $n$ is even or odd, and similarly for $p$ and $q$, the term is, in the four cases respectively,

$$
\begin{aligned}
& =2 \alpha^{m} \beta^{2 n} \quad\left(\begin{array}{llll}
\gamma^{2 p} & \gamma^{\prime 2 q}+\gamma^{2 q} & \gamma^{\prime 2 p}
\end{array}\right), \\
& =2 \alpha^{m} \beta^{2 n+1}\left(\begin{array}{llll}
\gamma^{2 p} & \gamma^{\prime 2 q} & -\gamma^{2 q} & \gamma^{\prime 2 p}
\end{array}\right) \text {, } \\
& =2 \alpha^{m} \beta^{2 n} \quad\left(\gamma^{2 p+1} \gamma^{\prime 2 q+1}-\gamma^{2 q+1} \gamma^{\prime 2 p+1}\right) \text {, } \\
& =2 \alpha^{m} \beta^{2 n+1}\left(\gamma^{2 p+1} \gamma^{\prime 2 q+1}+\gamma^{2 q+1} \gamma^{\prime 2 p+1}\right) .
\end{aligned}
$$

The second, third, and fourth expressions contain the factors

$$
\beta\left(\gamma^{2}-\gamma^{\prime 2}\right), \quad \gamma \gamma^{\prime}\left(\gamma^{2}-\gamma^{\prime 2}\right), \quad \beta \gamma \gamma^{\prime},
$$

respectively; and the first expression as it stands, and the other three divested of these factors respectively are rational functions of $\alpha, \beta^{2}, \gamma^{2}, \gamma^{\prime 2}$, that is, they are rational functions of $m, n, e, h$. But the omitted factors $\beta\left(\gamma^{2}-\gamma^{\prime 2}\right), \gamma \gamma^{\prime}\left(\gamma^{2}-\gamma^{\prime 2}\right)$, $\beta \gamma \gamma^{\prime},=2 n h\left(1+e^{2}\right), 2 h^{2} e\left(1+e^{2}\right)$, nhe $\left(1+e^{2}\right)$ are rational functions of $n, h, e$; hence each of the original four expressions is a rational function of $m, n, h, e$; and the entire function

$$
\phi\left(a, a_{1}, a_{2}, a_{3}\right)+\phi\left(a_{1}, a_{2}, a_{3}, a\right)+\phi\left(a_{2}, a_{3}, a, a_{1}\right)+\phi\left(a_{3}, a, a_{1}, a_{2}\right)
$$

is a rational function of $m, n, h, e$.

Replacing $\alpha, \beta, \gamma, \gamma^{\prime}$ by their values, the roots of the quartic equation are

$$
\begin{aligned}
& m+n \sqrt{ }\left(1+e^{2}\right)+\sqrt{ }\left[h\left(1+e^{2}+\sqrt{ }\left(1+e^{2}\right)\right)\right], \\
& m-n \sqrt{ }\left(1+e^{2}\right)+\sqrt{ }\left[h\left(1+e^{2}-\sqrt{ }\left(1+e^{2}\right)\right)\right], \\
& m+n \sqrt{ }\left(1+e^{2}\right)-\sqrt{ }\left[h\left(1+e^{2}+\sqrt{ }\left(1+e^{2}\right)\right)\right], \\
& m-n \sqrt{ }\left(1+e^{2}\right)-\sqrt{ }\left[h\left(1+e^{2}-\sqrt{ }\left(1+e^{2}\right)\right)\right] .
\end{aligned}
$$

And I stop to remark that taking $m, n, e, h=-\frac{1}{4},+\frac{1}{4}, 2,-\frac{1}{8}$ respectively, the roots are

$$
\begin{aligned}
& -\frac{1}{4}+\frac{1}{4} \sqrt{5}+\sqrt{ }\left[-\frac{1}{8}(5+\sqrt{5})\right], \\
& -\frac{1}{4}-\frac{1}{4} \sqrt{5}+\sqrt{ }\left[-\frac{1}{8}(5-\sqrt{5})\right], \\
& -\frac{1}{4}+\frac{1}{4} \sqrt{5}-\sqrt{ }\left[-\frac{1}{8}(5+\sqrt{5})\right], \\
& -\frac{1}{4}-\frac{1}{4} \sqrt{5}-\sqrt{ }\left[-\frac{1}{8}(5-\sqrt{5})\right],
\end{aligned}
$$


viz. these are the imaginary fifth roots of unity, or roots $r, r^{2}, r^{4}, r^{3}$ of the quartic equation $x^{4}+x^{3}+x^{2}+x+1=0$; which equation, as is well known, has the group $r r^{2} r^{4} r^{3}, r^{2} r^{4} r^{3} r, r^{4} r^{3} r r^{2}, r^{3} r r^{2} r^{4}$. form

Reverting to Abel's expression for $x$, and writing this for a moment in the the quintic equation in $x$ is

$$
x=c+p+s+r+q \text {, }
$$

$$
\begin{aligned}
0 & =(x-c)^{5} \\
& +(x-c)^{3} \cdot-5(p r+q s) \\
& +(x-c)^{2} \cdot-5\left(p^{2} s+q^{2} p+r^{2} q+s^{2} r\right) \\
& +(x-c) \cdot-5\left(p^{3} q+q^{3} r+r^{3} s+s^{3} p\right)+5\left(p^{2} r^{2}+q^{2} s^{2}\right)-5 p q r s \\
& +(x-c)^{0} \cdot-\left(p^{5}+q^{5}+r^{5}+s^{5}\right) \\
& +5\left(p^{3} r s+q^{3} s p+r^{3} p q+s^{3} q r\right) \\
& -5\left(p^{2} q^{2} r+q^{2} r^{2} s+r^{2} s^{2} p+s^{2} p^{2} q\right) .
\end{aligned}
$$

If we substitute herein for $p, q, r, s$ their values, then, altering the order of the terms, the final result is found to be

$$
\begin{aligned}
& 0=(x-c)^{5} \\
& +(x-c)^{3} \cdot-5\left(A A_{2}+A_{1} A_{3}\right) a a_{1} a_{2} a_{3} \\
& +(x-c)^{2} .-5\left(A^{2} A_{1} a_{2} a_{3}+A_{1}{ }^{2} A_{2} a_{3} a+A_{2}{ }^{2} A_{3} a a_{1}+A_{3}{ }^{2} A a_{1} a_{2}\right) a a_{1} a_{2} a_{3} \\
& +(x-c) .-5\left(A^{3} A_{3} a_{1} a_{2}{ }^{2} a_{3}+A_{1}{ }^{3} A a_{2} a_{3}{ }^{2} a+A_{2}{ }^{3} A_{1} a_{3} a^{2} a_{1}+A_{3}{ }^{3} A_{2} a a_{1}{ }^{2} a_{2}\right) a a_{1} a_{2} a_{3} \\
& +5\left(A^{2} A_{2}{ }^{2}+A_{2}{ }^{2} A_{3}{ }^{2}-A_{1} A_{2} A_{3} A\right)\left(a a_{1} a_{2} a_{3}\right)^{2} \\
& +(x-c)^{0} .-\left(A^{5} a_{1} a_{2}{ }^{3} a_{3}{ }^{2}+A_{1}{ }^{5} a_{2} a_{3}{ }^{3} a_{1}{ }^{2}+A_{2}{ }^{5} a_{3} a^{3} a_{1}{ }^{2}+A_{3}{ }^{5} a a_{1}{ }^{3} a_{2}{ }^{2}\right) a a_{1} a_{2} a_{3} \\
& +5\left(A^{3} A_{1} A_{2} a_{2} a_{3}+A_{1}{ }^{3} A_{2} A_{3} a_{3} a+A_{2}{ }^{3} A_{3} A a a_{1}+A_{3}{ }^{3} A A_{1} a_{1} a_{2}\right)\left(a a_{1} a_{2} a_{3}\right)^{2} \\
& -5\left(A^{2} A_{3}{ }^{2} A_{2} a_{1} a_{2}+A_{1}{ }^{2} A^{2} A_{3} a_{2} a_{3}+A_{2}{ }^{2} A_{1}{ }^{2} A a_{3} a+A_{3}{ }^{2} A_{2}{ }^{2} A_{1} a a_{1}\right)\left(a a_{1} a_{2} a_{3}\right)^{2} \text {; }
\end{aligned}
$$

viz. considering herein $A, A_{1}, A_{2}, A_{3}$ as standing for their values

$$
K+K^{\prime} a+K^{\prime \prime} a_{2}+K^{\prime \prime \prime} a a_{2}, \& c .
$$

respectively, each coefficient is a function of $a, a_{1}, a_{2}, a_{3}$, which is unaltered by the cyclical change of these values and therefore is a rational function of

$$
m, n, e, h, K, K^{\prime}, K^{\prime \prime}, K^{\prime \prime \prime} .
$$

